SHORT REPORT

\title{
Low dose of snake antivenom is as effective as high dose in patients with severe neurotoxic snake envenoming
}

\author{
R Agarwal, A N Aggarwal, D Gupta, D Behera, S K Jindal
}

Emerg Med J 2005;22:397-399. doi: 10.1136/emj.2004.020727

The objective of this study was to evaluate the effects of two different dosage protocols on the outcome of patients with severe neurotoxic snake envenoming, using a retrospective analysis of patients admitted with a diagnosis of severe neurotoxic snake bite over a 4 year period. In the study, 55 snake bite victims requiring ventilatory support for severe neurotoxic envenoming received either $150 \mathrm{ml}$ of polyvalent snake antivenom (SAV) (low dose SAV group, $n=28$ ) or $100 \mathrm{ml}$ of SAV at presentation followed by $100 \mathrm{ml}$ every 6 hours until recovery of neurological manifestations (high dose group, $n=27$ ). The median dose of SAV in the high dose group was $600 \mathrm{ml}$ (range 300 to 1600). The duration of mechanical ventilation in the low dose group (median 47.5 hours; range 14 to 248 ) was similar to that in the high dose group (median 44 hours; range 6 to 400). The mean (SD) duration of intensive care unit stay was similar in the two groups. There were three deaths in the high dose group; two patients in the low dose group had neurological sequelae. All other patients improved, had no residual neurological deficit, and were discharged. We conclude that there is no difference between a protocol using lower doses of SAV and one with higher doses in the management of patients with severe neurotoxic snake envenoming.

$\mathrm{S}$ nake envenoming is a common medical emergency encountered in the tropics, and an estimated 35000 to 50000 people die of snake bite every year in India. ${ }^{1}$ The bites of elapid snakes (cobras) cause predominantly neurotoxicity, which manifests as paralysis of ocular, bulbar, limb and respiratory muscles. ${ }^{2}$ The management of these patients includes ventilatory support and administration of snake antivenom (SAV).

The dose of SAV required in the management of severe neurotoxic snake envenoming should be based on measuring serial venom concentrations in patients and determining when free venom concentrations are undetectable; however, this is rarely clinically feasible. In the absence of any definite data, ${ }^{4}$ most recommendations are based on mouse assays, where the lethal dose is estimated to be around $120 \mathrm{mg}$ of cobra venom and $60 \mathrm{mg}$ of krait venom. ${ }^{5}$ The amount of venom neutralised by $1 \mathrm{ml}$ of SAV is approximately $0.6 \mathrm{mg}$ and $0.45 \mathrm{mg}$ for cobra and krait respectively. Thus, empirically, the total SAV requirement for otherwise fatal cobra and krait bites is 200 and $134 \mathrm{ml}$ respectively. However, this may not be true for human bites, as the exact total amount of venom injected by the snake at the time of bite is variable depending on the species and size of the snake, the mechanical efficiency of the bite, whether one or two fangs penetrated the skin, and whether there were repeated strikes. There is no consensus on the dose of SAV required in the management of snake bite, and selection of a particular dose of SAV is a controversial issue.

The aim of this study was to assess the effects of two different dosage protocols on the outcomes of patients with severe neurotoxic snake envenoming.

\section{MATERIALS AND METHODS}

The study was a retrospective analysis of patients treated at the respiratory intensive care unit (RICU) of this institute over the last 4 years. Patients with severe neurotoxic envenoming (defined as requirement of mechanical ventilation for ventilatory failure) were included in the study. Snake envenoming was diagnosed based on the history of snakebite and neurotoxic manifestations, presence of fang marks, presence of local manifestations such as swelling, cellulitis, and blister formation, or if the dead snake was brought in for identification.

Detailed history, findings of physical examination including site of bite, local reaction at the bite site and systemic features, and records of investigations including arterial blood gases, serum biochemistry, complete blood count, and coagulation profile were noted. Details of the dosage and schedules of SAV were recorded. Based on the prevalent practice in the RICU at different times, the patients could be divided into two groups. Group I (high dose group; before June 2002) received $100 \mathrm{ml}$ of polyvalent SAV (Haffkine Institute, Mumbai, India) at presentation followed by $100 \mathrm{ml}$ every 6 hours (no upper limit) until recovery of neurological manifestations. Group II (low dose group; July 2002-July 2004) received $100 \mathrm{ml}$ of SAV at presentation followed by $50 \mathrm{ml} 6$ hours later. Neostigmine or other cholinesterase inhibitors were not administered to any victim.

The primary outcome measures were the duration of mechanical ventilation (time in hours from the onset of intubation to extubation) and the duration of RICU stay. Mortality and other complications during the RICU stay were also recorded.

Continuous variables were compared using unpaired Student's $t$ test and categorical variables using $\chi^{2}$ test. Survival curves were constructed to compare the duration of mechanical ventilation in the two groups using KaplanMeier analysis and log rank test.

\section{RESULTS}

During the study period, 55 patients were admitted, 27 in the high dose and 28 in the low dose group (table 1). Snake bite was confirmed by patient report, correlation between clinical manifestations, and recognition of snakes by patients and bystanders. Only three dead snakes were brought for identification: two kraits and one cobra. The exact species was not identified because of the non-availability of venom specific enzyme immunoassay.

Abbreviations: RICU, respiratory intensive care unit; SAV, snake antivenom 
Table 1 Baseline characteristics and outcome of patients in low dose and high dose snake antivenom group

\begin{tabular}{|c|c|c|}
\hline & Low dose group & High dose group \\
\hline Number of patients & 28 & 27 \\
\hline Male/female & $25 / 3$ & $23 / 4$ \\
\hline Mean (SD) age, years & $28.3(14.5)$ & $32.2(14.8)$ \\
\hline $\begin{array}{l}\text { Delay in presentation } \\
\text { after bite, hours }\end{array}$ & $6.9(5.6)$ & $6.5(4.5)$ \\
\hline $\begin{array}{l}\text { Local signs and } \\
\text { symptoms }\end{array}$ & $3(10.7)$ & $4(14.8)$ \\
\hline Snakebite fang marks & $22(78.6)$ & $21(77.8)$ \\
\hline $\begin{array}{l}\text { Median SAV dose } \\
\text { (range), ml }\end{array}$ & 150 & 600 (300 to 1600$)$ \\
\hline $\begin{array}{l}\text { Median extubation } \\
\text { time (range), hours }\end{array}$ & 47.5 (14 to 248 ) & 44 (12 to 400$)$ \\
\hline $\begin{array}{l}\text { Mean (SD) RICU stay, } \\
\text { days }\end{array}$ & $3.9(2.0)$ & $3.9(3.0)$ \\
\hline Mortality & 0 & $3(11.1)$ \\
\hline
\end{tabular}

At admission, all patients had ptosis, dysphagia, diplopia, dysphonia, and evidence of hypercapnic respiratory failure $\left(\mathrm{PaO}_{2}<60 \mathrm{mmHg}\right.$ and $\left.\mathrm{PaCO}_{2}>45 \mathrm{mmHg}\right)$. None of the patients had any evidence of coagulopathy or renal failure at admission. The median dose of SAV in the high dose group was $600 \mathrm{ml}$ (range 300 to 1600); all patients in the low dose group received $150 \mathrm{ml}$ (table 1). Four patients in the low dose group and three in the high dose group showed hypersensitivity reactions to SAV, and were treated with promethazine and hydrocortisone. There was no episode of anaphylaxis. The mean duration of RICU stay was similar in the two groups (table 1). The median time to extubation in the high dose and low dose SAV groups was 44 and 47.5 hours respectively, and was not significantly different in the two groups using log rank test (fig 1). Two patients in the low dose group had ventilator associated pneumonia and one patient each in both groups had stress related minor gastrointestinal bleeding. There were three deaths in the high dose group. These three patients had probably sustained irreversible neurological damage before reaching the hospital and succumbed to complications related to ventilator associated pneumonia and sepsis. Two patients in the low dose group had residual neurological deficit in the form of extrapyramidal symptoms and myoclonic seizures at the time of discharge from hospital. All other patients improved and were discharged.

\section{DISCUSSION}

Neurotoxic snake envenoming is one of the most important causes of snake bite fatality ${ }^{1}$ and is mainly due to the Elapidae family, which includes the Naja and Bungarus

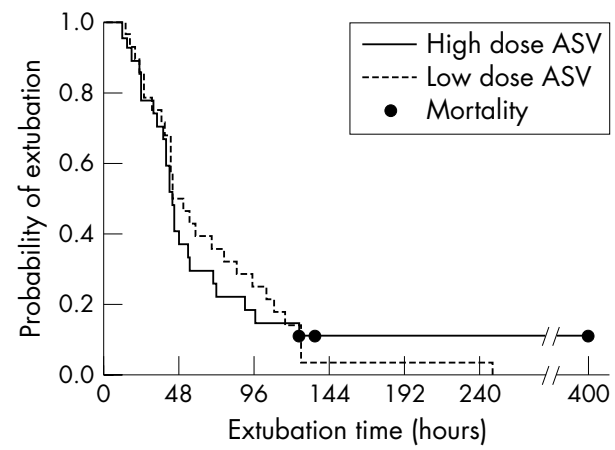

Figure 1 Extubation times in the low dose and high dose snake antivenom (SAV) groups. The duration of mechanical ventilation was similar in the two groups (log rank test). species, commonly referred to as the cobras and kraits respectively. Neuromuscular paralysis in snake bite occurs as a result of blockade of neuromuscular transmission. Toxins from cobra venom predominantly act post-synaptically, whereas those of krait venom mainly act presynaptically; however, most snake venoms contain both pre-synaptic and post-synaptic neurotoxins. ${ }^{26}$ SAV is a specific antidote to snake venom actions, and is the only effective treatment for neutralisation of venom that has entered the circulation.

Although SAV has been used for many years, there is no universal consensus in many countries on the optimal dose and protocol of its administration. Theoretically, it would appear that patients with more severe envenoming need higher doses of SAV for effective neutralisation of circulating snake venom. In the absence of any recommendations for management of patients with severe neurotoxic envenoming, higher doses have been previously used in the hope of early recovery. ${ }^{4}$ However, because of the high cost and limited availability of SAV, and reports of patients with severe envenoming recovering without the use of $\mathrm{SAV}^{8}$, there was a change in dosage protocols from high to low. Moreover, large doses of SAV may not cause any improvement in patients with presynaptic neurotoxicity, which is probably due to the irreversible effects of these toxins (although the clinical significance of pre-synaptic inhibition is difficult to assess ${ }^{6}$ ). The antivenom is effective only if given early enough to neutralise the venom in the circulation, prior to the neurotoxins reaching their target site, and thus prevent further development of neurotoxicity. Therefore, the use of large doses late in the course of envenoming is unlikely to be effective.

A few studies have addressed the issue of SAV dosage, but did not specifically include patients with severe neurotoxic envenoming, and outcome measures, especially the details on the duration of mechanical ventilation, were not particularly

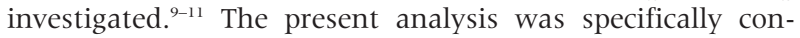
ducted to evaluate if usage of a higher dosage of SAV offered any significant clinical advantage over a lower dose in patients with severe neurotoxic envenomation. We found no significant difference in the outcome between the two groups.

What are the advantages of using a lower dose of SAV? The economic significance of using low doses of SAV is obvious. Each $10 \mathrm{ml}$ vial of SAV in India costs 400 rupees (approximately \$9), and use of lower dosages could translate into huge savings to the patient and the community. In addition, there is an increasing shortage of SAV in several developing countries, ${ }^{12}$ and an important incentive for a regulated dosing protocol would be to prevent crisis of SAV availability and supply. Finally, the incidence of early anaphylaxis and late serum sickness-type reactions, which is related to the dose of $\mathrm{SAV},{ }^{4}{ }^{13}$ can be decreased.

Our study has certain limitations. Firstly, the exact characterisation of the specific species was not performed by venom specific enzyme immunoassay, as this test is not routinely available. Secondly, the results of our study do not suggest that it should be applied to all snake bites. However, it definitely does suggest that lower doses of SAV may be as efficacious as higher doses in severe neurotoxic snake envenoming. Finally, laboratory assessments of SAV potency ${ }^{14}$ should serve as a guide to the use of antivenom dose in a particular region, and the onus thus rests on the clinicians to involve their patients in clinical trials to confirm the dose of SAV needed in their region. ${ }^{15}$

In conclusion, within the limitations of the present study, there is no difference between a protocol employing lower doses of SAV to higher dose in the management of patients with "unselected" severe neurotoxic snake envenoming. 


\section{ACKNOWLEDGEMENTS}

The authors thank Dr Balamugesh, Dr C Reddy, and Dr A Nath for their help while carrying out the study.

\section{Authors' affiliations \\ R Agarwal, A N Aggarwal, D Gupta, D Behera, S K Jindal, Department of Pulmonary Medicine, Postgraduate Institute of Medical Education and Research, Chandigarh, India \\ Competing interests: none declared}

Correspondence to: Dr R Agarwal, Department of Pulmonary Medicine, Postgraduate Institute of Medical Education and Research, Sector-12, Chandigarh 160012, India; drritesh1@rediffmail.com

Accepted 6 December 2004

\section{REFERENCES}

1 Warrell DA and international panel of experts. WHO/SEARO Guidelines for the clinical management of snake bites in the Southeast Asian region. Southeast Asian J Trop Med Public Health 1999;30(suppl 1):1-85.

2 Lewis RL, Gutmann L. Snake venoms and the neuromuscular junction. Semin Neurol 2004;24:175-9.

3 Riviere G, Choumet $\mathrm{V}$, Audebert $\mathrm{F}$, et al. Effect of antivenom on venom pharmacokinetics in experimentally envenomed rabbits: toward an optimization of antivenom therapy. J Pharmacol Exp Ther 1997;281:1-8.
4 Warrell DA. Animal toxins. In: Cook GC, Zumla A, eds. Manson's tropical diseases. London: WB Saunders, 2003:581-618.

5 Bawaskar HS. Snake venom and antivenom: Critical supply issues. J Assoc Physicians India 2004;52:11-13.

6 Harris JB. Snake venoms in science and clinical medicine 3. Applied immunology in snake venom research. Trans $R$ Soc Trop Med Hyg 1989;83:745-47

7 Agrawal PN, Aggarwal AN, Gupta D, et al. Management of respiratory failure in severe neuroparalytic snake envenomation. Neurol India 2001:49:25-8.

8 Bomb BS, Roy S, Kumawat DC, et al. Do we need anti-snake venom for management of elapid ophitoxemia? J Assoc Physicians India 1996;44:31-3.

9 Lalloo DG, Trevett AJ, Korinhona A. Snake bites by the Papuan taipan (Oxyuranus scutellatus canni): Paralysis, hemostatic and electrocardiographic abnormalities and effects of antivenom. Am J Trop Med Hyg 1995;52:525-31.

10 Tariang DD, Philip PJ, Alexander G, et al. Randomized controlled trial on the effective dose of anti-snake venom in cases of snake bite with systemic envenomation. J Assoc Physicians India 1999:47:369-72.

11 Paul V, Pratibha S, Prahlad KA, et al. High-dose anti-snake venom versus lowdose anti-snake venom in the treatment of poisonous snake bites - a critical study. J Assoc Physicians India 2004;52:14-17.

12 Warrell DA. Crisis in snake anti-venom supply for Africa. Lancet 2000:356:2104.

13 Theakston RDG, Fan HW, Warrell DA, et al. Am J Trop Med Hyg 1992;47:593-604.

14 Smalligan R, Cole J, Brito N, et al. Crotaline snake bite in the Ecuadorian Amazon: randomised double blind comparative trial of three South American polyspecific antivenoms. Br Med J 2004;329:1 129-36.

15 Theakston RDG, Warrell DA, Griffiths E. Report of a WHO workshop on the standardization and control of antivenoms. Toxicon 2003;41:541-57.
COMMENTARY

\section{Snake antivenom research: the importance of case definition}

\section{G K Isbister}

$\mathrm{S}$ nake envenoming is a major public health issue in the rural tropics, with large numbers of envenomings and deaths. ${ }^{12}$ However, there continues to be limited evidence on the clinical features, epidemiology, and treatment of these patients. ${ }^{12}$ In some parts of the world, there is a continuing shortage of antivenom. ${ }^{3}$ Agarwal et al ${ }^{4}$ attempt to answer a pertinent clinical question in their region. The study aims to justify a reduced dose of antivenom (thus reducing cost of treatment) in their hospital and surrounding regions. However, while the study provides useful insight into the issues of snakebite management in the tropics, there are a number of problems with the design, and the study cannot be generalized to other parts of the world. The appropriate design for the study would be a randomised, controlled trial (RCT), but these are not often undertaken because of the difficulties with RCT in this setting and the resources needed to measure venom concentrations in envenomed patients.

RCTs of antivenom are difficult to undertake for a number of reasons. The clear efficacy in many cases makes it unethical to perform placebo RCTs. It is possible to perform comparison RCTs of different antivenoms or comparison of different dosing regimens. ${ }^{5-7} \mathrm{~A}$ more significant problem is making sure that controlled trials include only definite cases of snake envenoming, and that a known and relatively homogenous type and severity of envenoming is being studied, so that results are not biased. An RCT of scorpion antivenom in Tunisia, ${ }^{8}$ which showed no benefit of antivenom, has been criticized because it included only a small number of severely envenomed patients, the group most likely to benefit from antivenom. ${ }^{9}$

Clinical toxinology has been plagued by poor case definition, ${ }^{10}$ leading to the erroneous association of many clinical syndromes with particular venomous or poisonous animals. ${ }^{11}$ This creates problems when undertaking clinical trials in clinical toxinology because if the study includes other than definite cases, it is difficult to determine what is the treatment effect. This is a significant problem in the study by Agarwal et al. Their study included any patients with "severe neurotoxic envenoming" who required mechanical ventilation, and not snake bites that caused neurotoxicity (requiring mechanical ventilation) and for which their was a definite species identifcation. This meant that the study included two very different types of snakes (cobras and kraits) without being able to identify within the study which patients were bitten by which snake.

The response of neurotoxicity to snake antivenom is dependent on the type of neurotoxins the snakes possess. ${ }^{12}$ Cobra venom contains mainly post-synaptic neurotoxins, which have a curare-like effect and can be reversed by snake antivenom after clinical effects have developed. Conversely, krait venom contains many presynaptic neurotoxins, which are not reversible once paralysis has developed and so respond poorly to delayed antivenom. ${ }^{12}$ The study by Agarwal et al therefore contains an unknown proportion of cases that may respond well to antivenom (cobra bites) and an unknown proportion that are unlikely to respond to antivenom (krait bites). In three cases, dead snakes were collected, demonstrating that both snake types were included in the study. Because their study was not randomized and followed a "before and after" design, an increase in the proportion of cobra bites over time could have resulted in the low dose ("after") group being inherently more likely to respond to antivenom than the high dose ("before") group. Even if the study was randomized, if both groups were predominantly krait bites and unlikely to benefit from antivenom, then there 
would be little difference between groups, but any beneficial effect of the higher antivenom dose for the smaller proportion of cobra bites would have been masked. Therefore, even in the setting of the authors' own hospital, their results should be seen only as a useful exploratory investigation or pilot study for future more rigorous study designs.

The reason that case definition is problematic in most studies is the difficulty involved in defining definite bites and establishing the exact species/ group involved. ${ }^{9}$ Snakes are not caught or collected in many cases, as seen in this report, although this study had a particularly low rate of snake capture ( 3 out of 55). The other method of snake identification is using an ELISA to detect snake venom in patient blood. ${ }^{13}$ One disadvantage of this method is that there may be significant cross reactivity, but this is unlikely to be a problem with distinguishing different genera of snakes such as cobras (Naja spp.) and kraits (Bungarus spp.). The other problem is the cost and access to such testing, as stated by Agarwal et al. However, there is a difference between "point of care" venom detection kits, such as those used in Australia, ${ }^{14}$ and the collection and storage of samples for research that can be sent to distant laboratories to be analysed. The latter is certainly possible in the rural tropics by collaboration with international research groups. This has been carried out in many parts of the developing world, ${ }^{13}$ including Sri Lanka, with similar snakes. ${ }^{15}$ The major advantage of ELISA is that serial venom concentrations can be quantified, which will allow an accurate determination of antivenom dose required. ${ }^{13}$ Such studies with definite case inclusion are desperately required to improve antivenom treatment for snake envenoming.

\section{ACKNOWLEDGEMENTS}

Thanks to $S$ Brown and B Currie for commenting on the manuscript.

Emerg Med J 2005;22:399-400.

doi: $10.1136 / \mathrm{emj} .2004 .022251$

Correspondence to: G K Isbister, Senior Research Fellow and Clinical Toxicologist Tropical Toxinology Unit, Charles Darwin University, Darwin and Newcastle Mater Hospital, Newcastle, Australia; gsbite@ ferntree.com

Accepted for publication 12 January 2005

Competing interests: none declared

\section{REFERENCE}

1 White J, Warrell D, Eddleston $M$, et al. Clinical toxinology-where are we now? I Toxicol Clin Toxicol 2003;41:263-76.

2 Chippaux J-P. Snake-bites: appraisal of the global situation. Bull World Health Organ 1998:76:515-24.

3 Theakston RDG, Warrell DA. Crisis in snake antivenom supply for Africa. Lancet 2000;356:2104.

4 Agarwal R, Aggarwal A N, Gupta D, Behera D, Jindal S K. Low dose of anti-snake venom is as effective as high dose in patients with severe neurotoxic snake envenoming. Emerg Med J 2005;22:397-9.

5 Smalligan R, Cole J, Brito N, et al. Crotaline snake bite in the Ecuadorian Amazon: randomised double blind comparative trial of three South American polyspecific antivenoms. BMJ 2004;329: 1129

6 Caovilla JJ, Barros EJ. Efficacy of two different doses of antilonomic serum in the resolution of hemorrhagic syndrome resulting from envenoming by Lonomia obliqua caterpillars: a randomized controlled trial. Toxicon 2004;43:81 1-18.

7 Ariaratnam CA, Sjostrom L, Raziek Z, et al. An open, randomized comparative trial of two antivenoms for the treatment of envenoming by Sri Lankan Russell"s viper (Daboia russelii russelii). Trans $\mathrm{R}$ Soc Trop Med Hyg 2001:95:74-80.

8 Abroug F, Elatrous S, Nouira S, et al. Serotherapy in scorpion envenomation: a randomised controlled trial. Lancet 1999;354:906-9.

9 Isbister GK, Graudins A, White J, et al. Antivenom treatment in arachnidism. J Toxicol Clin Toxicol 2003;41:291-300.

10 Isbister GK. Data collection in clinical toxinology: debunking myths and developing diagnostic algorithms. J Toxicol Clin Toxico 2002:40:231-7.

11 Isbister GK. Necrotic arachnidism: the mythology of a modern plague. Lancet 2004;364:549-53.

12 White J. Elapid snakes. In: Dart RC, ed. Medical toxicology. Philadelphia: Lippincott Williams \& Wilkins, 2004:1566-78.

13 Theakston RD. An objective approach to antivenom therapy and assessment of first-aid measures in snake bite. Ann Trop Med Parasitol 1997:91:857-65.

14 Jelinek GA, Tweed C, Lynch D, et al. Cross reactivity between venomous, mildly venomous, and non-venomous snake venoms with the Commonwealth Serum Laboratories Venom Detection Kit. Emerg Med Australas 2004; 16:459-64.

15 Theakston RD, Phillips RE, Warrell DA, et al. Envenoming by the common krait (Bungarus caeruleus) and Sri Lankan cobra (Naja naja naja): efficacy and complications of therapy with Haffkine antivenom. Trans $R$ Soc Trop Med Hyg 1990;84:301-8. 